\title{
Aunando Enfoques Teóricos: el Enfoque de Derechos y el Institucionalismo Centrado en Actores
}

Putting Together Theoretical Approaches:

The Human Rights Approach and Actor-Centered Institutionalism

\section{Gonzalo Olmedo}

Licenciado en Historia.

Doctorando en Política y Gobierno.

Universidad Católica

de Córdoba - CONICET

1421576@ucc.edu.ar
Fecha de recepción:

18.9.17

Fecha de aceptación:

23.2.18

\section{Resumen}

El siguiente trabajo propone articular paradigmas teóricos disímiles que a la vez provienen de distintas disciplinas para analizar políticas sociales. Concretamente, se intenta incorporar elementos conceptuales del institucionalismo centrado en actores de Scharpf (1997) - que a su vez se vale de elementos y herramientas de la teoría de la acción racional y la teoría de la acción cognitiva de Boudon (2003) - y utilizarlo en el análisis de políticas públicas que recepten, al menos en su diseño, el enfoque de derechos humanos. A tal efecto, se hará un estudio exploratorio de un programa social, el PROGRESAR, el cual cumple las condiciones para ser analizado con el marco de análisis propuesto.

Palabras clave: enfoque de derechos -institucionalismo centrado en actores - PROGRESAR políticas sociales - inclusión social - juventud -

\section{Abstract}

The following essay attempts to construct an articulation between two different theoretical paradigms stemming from diverse disciplines to analyze public policy. Specifically, the article aims to incorporate conceptual elements borrowed from Actor-Centered Institutionalism - which in turn ap- 
peals to elements and tools from rational choice theory and Boudon's cognitive action theory (2003) - and apply it to the analysis of human rights-based public policy. For that purpose, an exploratory survey of a social program will be conducted, PROGRESAR, which meets the conditions to be analyzed under the proposed theoretical framework.

Key-words: Human Rights Approach - Actor-Centered Institutionalism - PROGRESAR - social policies social inclusion - youth

\section{Introducción $^{1}$}

Las políticas públicas pueden ser analizadas desde diferentes marcos de análisis o teorías. Distintas disciplinas dentro de las ciencias sociales se proponen explicar fenómenos de acuerdo a enfoques diferentes que toman mayor o menor relevancia dependiendo, entre otros factores, del momento histórico o del objeto de estudio bajo análisis. En consecuencia, el surgimiento, desarrollo o cambio de las políticas públicas serán mejor o peor explicados de acuerdo a la fortaleza teórica o empírica del marco de análisis adoptado. Desde la ciencia política o la sociología, por ejemplo, el enfoque neoinstitucionalista, en sus distintas variantes, ha tenido cierta prominencia en las últimas décadas en lo que hace al análisis de políticas públicas propiamente dicho. Otras perspectivas más críticas o ligadas al paradigma constructivista, tienden a analizar a la política pública desde el punto de vista del análisis del discurso tanto de decisores como destinatarios, o desde categorías filosóficas que desafian las visiones positivistas y neopositivistas de la verdad y la realidad. Las ciencias jurídicas, en tanto, focalizan su análisis en la interpretación de la norma y si ésta se aboca a ciertos preceptos éticos o procedimentales que toda regulación debería adoptar.

La problemática radica en que si bien estas disciplinas tienen categorías conceptuales comunes y comparten el estudio de un mismo fenómeno social, muchas veces los distintos paradigmas teóricos carecen de articulación entre sí, y los mismos investigadores son reticentes a que una comunicación multidisciplinaria y receptiva entre distintas corrientes teóricas se concrete. Es cierto que en algunos casos la distancia entre ciertas teorías es inconmensurable (Roth Deubel, 2008), y tanto metodológica como epistemológicamente esta articulación resulta imposible. Pero sí existen casos en los que ciertos elementos o categorías teóricas pueden ser tomadas prestadas y aplicadas a objetos de estudio distintos. O incluso utilizar parte de un enfoque proveniente de otra disciplina para proveer de mayor poder explicativo a cierto fenómeno social. 
Esta comunicación entre paradigmas disímiles se ha producido de manera continua y en distintos grados, como es el caso del constructivismo, que se nutre de los aportes de la filosofia pos-estructuralista de Foucault y de la teoría crítica de Habermas, para analizar las políticas públicas desde el ángulo del análisis del discurso (Roth Deubel, 2008). Algo similar sucede entre disciplinas sociales con objetos de estudios distintos, como la economía, la sociología y la ciencia política, donde desde miradas epistemológicas diferentes, logran explicar un determinado fenómeno social de manera unánime y coherente. Nuestra propuesta, siguiendo a Cairney $(2013),{ }^{2}$ plantea algo similar: tomar algunas categorías conceptuales de marcos de análisis disímiles pero pasibles de ser complementarios entre sí, y aplicarlas a un determinado objeto de estudio desde una mirada multidisciplinaria.

En particular, la propuesta consiste en tomar elementos conceptuales del institucionalismo centrado en actores de Scharpf (1997) - que a su vez se vale de elementos y herramientas de la teoría de la acción racional y la teoría de la acción cognitiva de Boudon (2003) - y utilizarlo en el análisis de políticas públicas que recepten, al menos en su diseño, el enfoque de derechos humanos.

En la siguiente sección se discutirá en qué consiste el institucionalismo de Scharpf y cuáles son los elementos de su propuesta teórica que más útiles resultan para el análisis de políticas sociales en clave de derechos. También se hará una breve descripción sobre en qué consiste este enfoque. Posteriormente, se hará un breve análisis del tipo de políticas que por definición han adoptado el enfoque de derechos humanos: las transferencias monetarias condicionadas (TMC). A tal efecto, se hará un estudio exploratorio de un programa social, el PROGRESAR, el cual cumple las condiciones para ser analizado con el marco de análisis propuesto. El artículo concluye con algunas reflexiones finales.

\section{El Marco de Análisis: Políticas con Enfoque de Derechos analizados desde el Institucionalismo Centrado en Actores}

La razón principal que nos lleva a cierta heterodoxia epistemológica es que numerosas políticas sociales, en especial aquellas orientadas a combatir la pobreza, contienen un lenguaje de derechos. Por otro lado, los análisis econométricos o los estudios de impacto, no contemplan los principios esgrimidos desde el enfoque y tienden a reducir los resultados obtenidos a datos. Si bien se han concretado importantes avances en el análisis de las políticas públicas desde las ciencias jurídicas, la metodología misma de esta disciplina previene, al menos prima facie, producir herramientas que superen la mera descripción y permitan explicar ciertos efectos o resultados de la política. Así, al combinar la ciencia política con ciertos rasgos de las ciencias jurídicas, podemos utilizar las categorías conceptuales presentes en ciertos enfoques dentro de estas disciplinas, para luego proveer de un marco explicativo más rico del fenómeno en cuestión. 
A modo de ejemplo, tomamos al programa de transferencias monetarias condicionadas PROGRESAR que contiene, tanto en la norma como en la retórica del programa, el lenguaje de derechos humanos. El enfoque de derechos se nutre de varios principios provenientes del sistema internacional de derechos humanos para contrastar y determinar en qué medida cierta política se acerca a estos valores. Así, la gran mayoría de los análisis de políticas públicas que practica este enfoque se centra en indagar si el programa comulga con principios del mismo, o en qué manera el programa implementa los valores propuestos en su normativa. En la mayoría de los casos, estos análisis concluyen que existe un déficit de derechos que perjudica indefectiblemente a los destinatarios y que la política no cumple con el enunciado ni los objetivos de la norma (Abramovich 2006; Abramovich y Courtis 2006; Abramovich y Pautassi 2006; Jiménez Benitez 2007; Arcidiácono 2011; Etchichury y Álvarez 2015; Olmedo 2016). Tales análisis son sumamente valiosos ya que, por un lado, nos proveen de un modelo ideal que toda política social debería seguir, y por el otro, marcan un límite a los decisores de la política para prevenir el abuso retórico de un lenguaje en clave de derechos que sin duda genera deferencia en ámbitos sociales y académicos.

Pero para entender más a fondo la política y proveerle de mayor poder de explicación, es necesario ahondar en las causas de por qué, por ejemplo, una política social adopta (o no) para su diseño el lenguaje de derechos, ${ }^{3}$ o el contexto histórico de los decisores políticos para ampararse en tal o cual enfoque. Tal vez más importante aún es saber por qué, a pesar de las probables buenas intenciones de los actores políticos, los destinatarios no logran ser sujetos de derechos plenos. Para dar respuesta a tales interrogantes, consideramos necesario complementar el enfoque de derechos con un marco de análisis más explicativo como lo es el institucionalismo centrado en actores de Fritz Scharpf.

\section{El Institucionalismo Centrado en Actores}

La política pública es el resultado, entre otros elementos, de un diálogo entre distintos actores de la sociedad, tanto gubernamentales como no gubernamentales. Cada política pública se desarrolla dentro de un marco institucional que delimita las esferas de acción, interpelando actores y condicionando su comportamiento. Repetto (2009) define al marco institucional como "los modos en que se agregan o desagregan intereses, se procesan los conflictos, se negocian intercambios de recursos y percepciones del mundo,...constreñidos por un tejido de mecanismos históricamente conformados" (pág. 143). El nuevo institucionalismo, con sus diversas y ricas variantes, nos ayuda a comprender la realidad social al otorgarle nuevamente un rol elevado a las instituciones de diversos tipos, pero a la vez reconociendo el rol de los actores individuales y colectivos (con sus decisiones y preferencias) tanto en la formación misma de las instituciones cuanto en las transformaciones que éstas experimentan. Todas sus variantes asumen a las instituciones como conjuntos de reglas e incentivos que condicionan la racionalidad de los actores (individuales y colectivos), estableciendo un espacio político donde éstos pueden interactuar (Peters 2003). 
Más precisamente, las instituciones constriñen o corrompen la conducta humana; es decir, inducen a la realización de ciertos comportamientos y no otros (Immergut 1998).

Teniendo en cuenta el riesgo de caer en un exceso de simplificación, dividiremos a la teoría institucionalista en dos grandes corrientes: el institucionalismo histórico (IH) y el institucionalismo de la elección racional (IER). ${ }^{4}$ En el primero, el papel central lo cumplen las instituciones, que son definidas como "los procedimientos formales e informales, rutinas, normas y convenciones incrustadas en la estructura organizacional de la comunidad política"5 (Hall and Taylor 1996:6). Así, estas reglas institucionales afectan el comportamiento de los actores generando efectos sobre las políticas, proveyendo distintos grados de certidumbre acerca del comportamiento presente y futuro de los otros actores. Para esta corriente, la formación de la institución es decisiva ya que esas reglas originales perdurarán (a pesar que se flexibilicen, otra característica de este enfoque) y moldearán las posteriores políticas que se sigan.

En el institucionalismo de la elección racional, en cambio, individuo ocupa el centro de la escena y actúa racionalmente para obtener un beneficio personal. En esta corriente, las instituciones funcionan como conjuntos de reglas que moldean la conducta individual, pero los individuos reaccionan racionalmente ante los incentivos y restricciones generados por estas reglas (Peters 2003). Algunos teóricos del institucionalismo de la elección racional consideran a las estructuras formales como medios para satisfacer el fin de los individuos: la maximización de sus beneficios individuales ${ }^{6}$. En tanto, el origen de las instituciones está fundamentado en que éstas reducen los costos de transacción. Las instituciones surgen así con el fin de otorgar mayor previsibilidad a los actores.

A pesar de haber recibido varias críticas, entre ellas el hecho de ser un modelo de tipo deductivo, la incapacidad de falsear hipótesis en el sentido popperiano y el exceso de confianza en demasiados supuestos simplificadores (y poco realistas) para su correcta aplicación, existen aquellos que consideran que estas debilidades son, en efecto, "liberalizadoras". Estos límites permiten al investigador, entre otras cosas, producir resultados y conclusiones más contundentes (Shepsle 2005). Así y todo, desde sus comienzos, el IER ha sufrido un gran escrutinio desde sus propios defensores, dando respuestas a muchas de las críticas antes mencionadas. Una de las respuestas más elaboradas es la concepción de bounded rationality (racionalidad limitada).

La racionalidad limitada puede ser definida como los casos en que la racionalidad falla $y$, en consecuencia, existe un desajuste entre el entorno que rodea la toma de decisiones y las opciones de quien toma las decisiones (Jones 1999). El pionero de este enfoque es Herbert Simon (1957), quien sugiere que los seres humanos somos "aproximadamente" racionales. La idea es que los actores toman decisiones que son satisfactorias, pero no necesariamente óptimas. Así, los actores racionales carecen de suficiente información para 
tomar decisiones maximizadoras debido a varias razones: la maleabilidad del problema a resolver, el tiempo disponible para tomar la decisión y la capacidad cognitiva del agente tomando la decisión. Por su parte, Boudon (2003) en su "teoría de la acción cognitiva" sugiere que hay que ir más allá del concepto de racionalidad desarrollada por el IER clásico, clasificando a la racionalidad en dos tipos: la racionalidad evaluativa o axiológica y la racionalidad cognitiva. Esta última no debe, según el autor, reducirse a su forma descriptiva, sino que debe ser complementada por la primera. En consecuencia, al concepto de racionalidad de Simon, donde los individuos no toman el camino óptimo sino el satisfactorio, se le suma la noción de Boudon, donde si bien los objetivos de los actores tienden hacia un fin determinado -en congruencia con los postulados de la elección racional-, éstos no necesariamente deben ser instrumentales (racionalidad evaluativa). Esto le otorga al IER mayor flexibilidad, proveyendo mayor importancia al entorno y la (in)capacidad cognitiva del actor, acercándolo más con los otros institucionalismos antes descritos. Scharpf (1997) tomará nota de algunos de estos conceptos y los articulará, junto a la teoría de los juegos, a su propio enfoque: el institucionalismo centrado en actores.

Para Shepsle (2005), las instituciones son las formas de hacer las cosas de manera equilibrada. En otras palabras, si para esta corriente las instituciones son identificadas como las reglas del juego (North 1990; Ostrom 1986), cuando las reglas no son respetadas -es decir, cuando no están en equilibrio- la institución es considerada frágil. Esta idea toma mayor relevancia cuando incorporamos en el mix a la teoría de los juegos, a la que Scharpf $(1989,1990,1991,1997)$ suscribe y utiliza como herramienta explicativa para comprender los efectos que surgen de la interacción entre actores (ya sean individuales o colectivos). El objetivo final de su enfoque es aplicar la teoría de los juegos a la investigación empírica (en un intento de mezclar el neoinstitucionalismo con la teoría de los juegos) para explicar fenómenos sociales. Ante esta sinergia, el concepto de institución de Scharpf (1997) es más amplio, al considerarla como sistemas de reglas (tanto formales o legales como sociales) ${ }^{7}$ que estructuran cursos de acción que los actores pueden elegir.

El eclecticismo en el modelo de Scharpf se manifiesta no sólo en la adopción del concepto de racionalidad limitada o el tomar prestado la teoría de los juegos de la matemática, sino también al considerar importante tomar en cuenta supuestos normativos para determinar si una política es buena o no (más allá de si es exitosa o es un fracaso) ${ }^{8}$. Esto es relevante en nuestra línea de investigación ya que indagaremos las razones que llevan a los gobiernos a adoptar, por ejemplo, el enfoque de derechos o a los actores a tomar decisiones que afectan (o no) la completa recepción del enfoque de derechos en sus políticas públicas.

Asimismo, Scharpf (1997) utilizará la narrativa en aquellos casos en que los modelos analíticos no estén disponibles. Más precisamente, se trata de confeccionar una "pequeña lista de módulos explicativos más integrados y situados históricamente para intentar 
capturar la lógica característica de las interacciones de políticas empíricamente observables en contextos institucionales del mundo real" (32). ${ }^{9}$ En consecuencia, haremos uso de la historia para mejor comprender el contexto institucional en el que se desarrollan los procesos de decisión. ${ }^{10}$ Aquí también el enfoque de derechos es digno de analizar, ya que es parte del contexto histórico en el cual se desarrollan las políticas públicas bajo análisis. Es así como se pretende desarrollar una perspectiva integradora entre el actor y la estructura: el enfoque de redes de políticas públicas (policy networks).

Dentro de las redes de políticas públicas, bajo el análisis de la gobernanza (governance), se encuentra el institucionalismo centrado en actores desarrollado por Scharpf. En este esquema, los fenómenos sociales deben ser explicados como el resultado de actores que poseen intenciones, pero estas interacciones están estructuradas, y sus efectos moldeados por las características de los contextos institucionales en los que éstos ocurren (Scharpf 1997). Las instituciones afectan no sólo las percepciones, preferencias y capacidades que tienen los actores, sino también las formas en que éstos interactúan (Zurbriggen 2006). Aunque las instituciones condicionan el comportamiento de los actores no lo determinan, ya que éstos poseen un amplio margen de decisión. Las instituciones funcionan, en consecuencia, como una forma de obtener previsibilidad, proveyendo de una invaluable fuente de información para el decisor, especialmente en relación a la conducta de los otros actores en una determinada arena política.

A partir de lo expresado anteriormente, Scharpf (2007) plantea un modelo que incorpora una serie de categorías analíticas teniendo en cuenta los conceptos de actor e institución. El autor propone la lógica situacional en la que "cada política pública fija un sistema de interacción sociopolítica estableciendo recursos a disposición de los actores, una estructura de oportunidades en específicos marcos institucionales (institucional settings) que hacen más o menos probable el desarrollo de determinados modos de interacción" (Zurbriggen 2006:10). ${ }^{11}$ Cabe recordar que se está en busca de un enfoque o, más bien, de una teoría de rango o alcance medio cuya aplicación está limitada a un espacio y tiempo determinado y que provea los lineamientos para la búsqueda de explicaciones. Las categorías desarrolladas hasta aquí con sus respectivas relaciones entre sí se encuentran resumidas en el cuadro siguiente: 


\section{El Enfoque de Derechos Humanos}

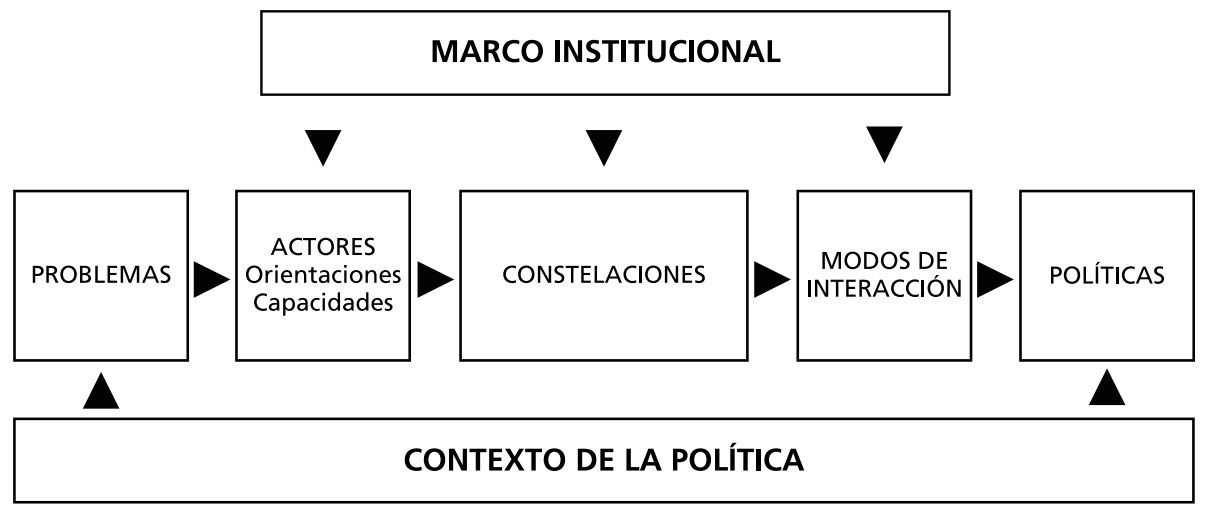

Fuente: Scharpf (1997)

Con esta perspectiva de las políticas públicas, incorporamos el enfoque de derechos. Este enfoque se centra en dos dimensiones: por un lado, reafirmar la importancia del principio de interdependencia que existe entre los derechos, es decir, cómo los derechos políticos y civiles están interrelacionados y dependen de los derechos económicos, políticos y sociales para su plena realización; y por el otro, asignar al estado un rol positivo como garante de derechos positivos hacia los ciudadanos.

El desarrollo del Derecho Internacional de los Derechos Humanos y su incidencia en los sistemas jurídicos nacionales han impulsado una evolución del derecho hacia una función no sólo de limitar a los estados en la comisión de violaciones, sino también de promocionar, proteger y ser responsables de la plena realización de los derechos humanos. Se busca asegurar el cumplimiento de esta función a través de mecanismos públicos (Poder Judicial, Defensoría del Pueblo) o privados (ONGs) de responsabilidad o de garantías (Abramovich 2006). Estos mecanismos institucionales y sociales limitan algunas acciones del estado a la vez que tutelan otras, facilitando la aplicación de principios de políticas públicas que respeten el reconocimiento de la integridad de los derechos humanos y su acceso a los sectores excluidos.

Desde esta perspectiva, no es correcta la concepción que considera a los derechos de primera generación como libertades negativas, mientras que los de segunda y tercera generación establecen libertades positivas. Tanto los derechos políticos y civiles como los económicos, sociales y culturales establecen obligaciones positivas y negativas, articuladas entre sí. Por ello, mejor que una categorización taxativa de derechos, Abramovich prefiere una conceptualización basada en un continuum de derechos (Abramovich 2004, 2006;Abramovich y Pautassi 2006), donde no se trata del tipo de derecho sino del peso simbólico que cada uno de estos tiene en cuanto a obligaciones positivas o negativas por parte del estado. 
Existe un creciente consenso en América Latina de pensar a las políticas públicas, especialmente las políticas sociales, como obligaciones estatales para hacer efectivo el cumplimiento de los derechos humanos (Abramovich y Pautassi, 2006). Bajo este esquema, se intenta dejar atrás la idea de las políticas públicas como paliativas de las "externalidades" del mercado, donde un grupo reducido de ciudadanos (aquellos que no sintieron los beneficios del llamado "efecto derrame") son meros beneficiarios de planes sociales focalizados. En cambio, un enfoque de derechos considera a las personas como titulares plenos de derecho o mejor, "sujetos de derecho". Es decir, se considera que "el primer paso para otorgar poder a los sectores excluidos es reconocer que son titulares de derechos que obligan al estado" (Abramovich y Pautassi 2006:17). Podríamos definir al enfoque de derechos como la traducción de las normas y principios internacionales de derechos humanos en acciones de política pública particulares. No se trata de cumplimentar mandatos morales o políticos, sino de imponerle al estado obligaciones jurídicas y exigibles tomando como paradigma los tratados de derechos humanos.

Numerosos autores y organismos dan cuenta de disfuncionalidades que pueden generarse en el proceso de políticas sociales, como por caso la baja calidad de los servicios prestados, como consecuencia de invertir en la demanda y no en la oferta (Cacace y Albornoz 2012). Si el aumento de la demanda por estos servicios no va de la mano del aumento en la oferta, puede comprometerse la calidad global de la atención. Zibecchi (2008) ejemplifica este problema al mencionar que en contextos de extrema pobreza y marginalidad, la escuela sirve de lugar de contención social. Así, la política asistencial recarga de trabajo a la burocracia educativa y deteriora el oficio de enseñar. Por su parte, Pautassi (2013) explica que esos efectos devienen de la falta de articulación de las TMC con otras políticas públicas activas. La autora explica que las TMC se convierten en mega herramientas de política social que a pesar de respaldarse en un enfoque de derechos, no han logrado instalar una acción suficientemente inclusiva y sustentable de la protección social y de la seguridad social.

Este enfoque tiene como objetivo principal servir de guía y orientar la formulación, implementación y evaluación de las políticas públicas para que éstas incorporen principios directrices que no violen los derechos humanos. Esto es, dotar a las políticas de desarrollo de una conceptualización de principios aplicables jurídicamente. El enfoque de derechos sirve, así, como un marco conceptual que contempla los principios o estándares que toda política pública debe seguir. Algunos de estos principios son universalidad, igualdad ( $y$ no discriminación), justiciabilidad, canales de diálogo, capacidades estatales, progresividad, participación y razonabilidad (GTPSS, 2015). La evidencia nos muestra que muchos programas enmarcados bajo este enfoque incluyen los principios antes descritos en el proceso de diseño de la política. Sin embargo, la falla se produce en el momento de implementación de la política en cuestión. 


\section{Las TMC y el PROGRESAR}

Hacia fines de 1990, pero con mayor fuerza en el presente siglo, ${ }^{12}$ los gobiernos de América Latina implementaron una variedad de programas sociales llamados de TMC. Esto se debe, en parte, al giro de la región hacia una "nueva izquierda", la que por un lado adopta políticas pro mercado, y por el otro, dirige sus recursos fiscales hacia un incremento significativo del gasto social. Coadyuvan en su momento una bonanza financiera asociada al precio de las materias primas y un crecimiento sostenido de las economías en desarrollo.

Los TMC son mecanismos de transferencia de recursos hacia los sectores en condiciones de exclusión (social, económica, cultural) a cambio de que la población incluida como beneficiaria siga un curso de acción específico (Bastagli 2009). Dichos programas han tenido entre sus principales objetivos el apoyo a las familias para mejorar sus condiciones de vida y la inversión en capital humano en materia de salud y educación. Se caracterizan por incorporar el lenguaje de derechos humanos, establecer condicionalidades y otros elementos modernizantes que los diferencia de sus antecesores. Concretamente, estas transferencias otorgan un beneficio económico en forma de efectivo a los destinatarios y éstos, como contrapartida, satisfacen una serie de condiciones que tienen como objetivo, en el corto plazo, reducir la pobreza, y en el largo, intentar eliminar la pobreza inter-generacional (Cacace y Albornoz 2012). Los programas TMC se enfocan en resolver problemas crecientes como la desocupación y la pobreza, y más específicamente, el acceso y la deserción escolar (muchas veces ligadas al trabajo infantil). ${ }^{13}$ En Latinoamérica, gobiernos de distintos signos políticos han implementado este tipo de políticas sociales; en muchos casos (como fue el caso del Programa Nacional de Becas Estudiantiles -PNBE- en Argentina), el diseño y la evaluación de estas políticas ha estado a cargo de los organismos internacionales de crédito, como el Banco Mundial.

Los programas de TMC han sido incorporados por la gran mayoría de los países de la región como la principal forma de política social para enfrentar la pobreza estructural, con la particularidad -entre otros aspectos- de asegurar un nivel básico de ingresos monetarios que promueve la autonomía del sujeto. En la literatura especializada se encuentran tres razones que explicarían la difusión de estos programas: tienen un costo presupuestario relativamente bajo (menos del 1\% del PBI en la mayoría de los casos); registran logros en la reducción de la pobreza, la desigualdad y la vulnerabilidad relativa; se observan progresos en los índices de educación y salud de los destinatarios (Cacace y Albornoz, 2012).

Existe un amplio repertorio de literatura académica que analiza la temática de las TMC. Los organismos internacionales de crédito, como el Banco Mundial o el Banco Interamericano de Desarrollo, analizan estas políticas desde la estructura de los programas midiendo desde la justificación económica para la implementación de las TMC hasta el tipo de condicionalidades o el diseño y ejecución de éstos en los países en desarrollo 
(Rodríguez Enríquez 2011). El objetivo principal de estos organismos es proveer de soluciones y recomendaciones para aquellos países que deseen implementarlos.

Existe, a su vez, una vasta literatura por parte de organismos gubernamentales y trabajos académicos abocada al estudio de impacto de estos planes. Desde esta perspectiva, se evalúan las transferencias condicionadas como instrumentos de disminución de la pobreza (Villatoro 2007; Fiszbein y Schady 2009), o como medios de reducción del ausentismo escolar y el índice de repitentes (Rawlings y Rubio 2003; Barrientos y Villa 2014). Algunos de estos autores también han medido la incidencia en la diversidad en el consumo de productos de primera necesidad (Rawlings y Rubio 2003; Fiszbein y Schady 2009), como así también en los índices de desigualdad (López-Calva y Lustig 2010; Levy y Schady 2013) y el trabajo infantil (Rosas 2007). Emparentado con esta modalidad de la política social, Hornes (2012) evalúa cómo el dinero recibido de las TMC ha operado en los procesos de sociabilidad económica; de este modo, los destinatarios no son vistos como receptores de planes sociales sino como agentes económicos activos.

En Argentina, Pautassi (2009), Abramovich (2006) y Arcidiácono (2008) han estudiado las TMC en función del enfoque de derechos y su aporte a las políticas públicas para enfrentar la pobreza. Asimismo, han trabajado la institucionalidad de las políticas sociales en las que se insertan estos programas. Más recientemente, Etchichury y Álvarez (2015) han analizado distintas políticas públicas nacionales bajo este enfoque, entre ellas el programa SUMAR, el Conectar Igualdad y el PROCREAR, utilizando un método jurídico-normativo.

\section{El programa PROGRESAR}

En Argentina, los TMC empiezan a implementarse de la forma antes descrita en 1996 a través del Programa Nacional de Becas Estudiantiles (PNBE), cuyo objetivo primario era la reinserción escolar -y no así la retención escolar- (Rosas, 2007).Varios programas sociales le siguen al PNBE, como el Programa Jefes y Jefas de Hogar Desocupados (2002), el Plan Familias para la Inclusión Social (2006) y la Asignación Universal por Hijo (2009), aunque el fin de éstos no sólo fue incrementar el capital humano, sino también disminuir la pobreza y la marginalidad. En los dos últimos planes, el estado argentino intentó sustituir la concepción de las personas como beneficiarios pasivos de la política social a sujetos o ciudadanos portadores de derechos (CELS 2007). En 2014 se instituyó el Programa de Respaldo a Estudiantes de Argentina o PROGRESAR (Dec. 84/2014), que distribuye un estipendio mensual destinado a jóvenes de entre 18 y 24 años que ni estudian ni trabajan. El antecesor de éste es el Programa Jóvenes con Más y Mejor Trabajo, que según el Decreto 84/2014, debe complementar al PROGRESAR. De acuerdo a ese decreto, el PROGRESAR tiene como objetivo

Generar oportunidades de inclusión social y laboral a través de acciones integradas que permitan capacitar a los jóvenes entre DIECIOCHO (18) y VEINTICUATRO 
(24) años de edad inclusive con el objeto de finalizar la escolaridad obligatoria, iniciar o facilitar la continuidad de una educación superior y realizar experiencias de formación $y$ /o prácticas calificantes en ambientes de trabajo. (Decreto No 84/2014:2).

Similar a otros programas, PROGRESAR se orienta a incrementar el capital humano de los beneficiarios con el fin de contribuir a su inserción en el mundo laboral y mejorar su capacidad de generar ingresos. El PROGRESAR es una suerte de continuación de la Asignación Universal por Hijo para la Protección Social (AUH) en poblaciones igualmente vulnerables, ya que incorpora a la población que deja de ser cubierta cuando llega a los dieciocho años de edad. Asimismo, se suma a otros programas vigentes de TMC, "expandiendo el sistema de transferencias directas orientadas a sostener los ingresos de las familias con jóvenes hasta 24 años a cargo" (Marzonetto y Aguirre 2014:12), en hogares con ingresos inferiores a tres salarios mínimos, vitales y móviles (SMVM). El programa tuvo como destinatarios a un universo de hasta 1,5 millones de jóvenes entre 18 y 24 años, es decir el 30\% de los 4 millones de jóvenes en ese rango etario (Mazzola 2014). A su vez, la política significa una inversión inicial correspondiente al $0,3 \%$ del PBI y hasta enero de 2018 estaba financiada por el Tesoro Nacional. ${ }^{14}$

El 30 de enero de 2018, a través del Decreto 90/2018 (modificatorio del Decreto N$^{\circ}$ 84/2014), el gobierno nacional relanzó el PROGRESAR, que pasó a llamarse Becas PROGRESAR. El nuevo programa mantiene los requisitos de acceso de su antecesor y su carácter universal. Por otro lado, los montos se actualizan y en algunos casos aumentan en términos reales, pero también lo hacen las exigencias para continuar siendo beneficiario. Por ejemplo, para mantener el beneficio, los estudiantes de la escuela secundaria deberán pasar de año, mientras que los alumnos terciarios y universitarios deberán aprobar la mitad más una de las materias del programa de estudios. Quienes logren aprobar todas las materias con un promedio de 8 o superior, pueden acceder a un premio a la excelencia a final del curso lectivo cuyo pago duplica el valor de la beca total anual.

Otro de los cambios relevantes es que el PROGRESAR pasa a la órbita del Ministerio de Educación (Decreto 90/2018). En principio, este cambio corresponde a dos razones principalmente. Por un lado, que los centros educativos puedan cruzar datos con el ministerio y hacer un seguimiento detallado del destinatario, es decir, que las escuelas y universidades estén al tanto de la condición de becario de sus alumnos. Por otro lado, se consolidan las becas universitarias existentes con el PROGRESAR, proveyendo distintas escalas de beneficios monetarios de acuerdo al nivel educativo que esté cursando el destinatario y privilegiando áreas o carreras que se consideren estratégicas, entre ellas las ingenierías, las Ciencias Exactas y Enfermería. 


\section{Aplicación del Marco de Análisis al PROGRESAR}

El marco institucional. En la perspectiva analítica de Scharpf (1997), el marco institucional determina las reglas del juego, lo que hace más o menos probable el desarrollo de determinados modos de interacción (Zurbriggen 2006). Es decir, son un elemento fundamental para entender los modos de interacción que se dan entre los actores, ya que limitan su accionar y los resultados deseables.

La Argentina posee un sistema presidencialista, donde gran parte del poder político recae en el Poder Ejecutivo. A su vez, la Constitución Nacional, a partir de la reforma de 1994, mediante el art. 75, inc. 22 incorpora y dota de rango constitucional a los Tratados Internacionales de Derechos Humanos. Esto significa no sólo que estos tratados tienen una jerarquía superior a las leyes, sino que el espíritu de las leyes debe coincidir con los principios vigentes en tales tratados.

Asimismo, la elaboración e implementación de las políticas sociales están a cargo del Poder Ejecutivo Nacional. A partir de la implementación de la AUH mediante Decreto de Necesidad y Urgencia (DNU), se sentó el precedente para la sanción de futuras políticas sociales salteando al Congreso de la Nación y valiéndose de los fondos de la Administración Nacional de Seguridad Social para solventar este tipo de políticas. Este procedimiento se alteró, en parte, a partir de la sanción de la ley de movilidad de la AUH en $2015^{15}$. Esta libertad de acción presupuestaria es facilitada también por la Ley de Emergencia Económica (Ley 25.561), sancionada en enero de 2002 y renovada hasta la fecha, que permite al Jefe de Gabinete reasignar recursos y partidas presupuestarias.

Se puede advertir cierta fragilidad en implementar políticas públicas por decreto (al no darse el debate en el Parlamento) así como también la falta de articulación de la política con otras políticas públicas relacionadas a la terminalidad educativa o la capacitación laboral, ya que sin el acompañamiento presupuestario y organizacional que un incremento masivo de la demanda de esos servicios supone, la calidad de los servicios prestados tendería a bajar. Este déficit de capacidades estatales, tanto desde el punto de vista administrativo cuanto en lo que respecta al financiamiento, supone un retroceso de la recepción de los principios del enfoque de derechos para con los beneficiarios.

Contexto de la política. El contexto o entorno tiene que ver con lo externo o macro que sucede en torno a la política. Es el contexto el que ayuda a orientar a los actores a saber cuándo y cómo actuar, proporcionando información sobre las demandas presentes y facilitando la imposición de determinados temas en la agenda, los cuales son pasibles de ser tomados por la pluralidad de actores que interactúan con la política. 
Como se mencionó anteriormente, desde hace más de dos décadas la gran parte de las políticas sociales en Argentina han tomado la forma de transferencias condicionadas y han sido más o menos focalizadas, lo que a su vez alimenta la trampa de la informalidad y la pobreza (Lo Vuolo, Barbeito et. al. 2004). Esto se traduce en desincentivos de los beneficiarios para dejar de percibir la ayuda estatal. Ante la repetida queja de la focalización, los organismos internacionales de crédito aportaron una versión mejorada de los programas, haciéndolos más universales, disminuyendo los requisitos de ingreso e incorporando un lenguaje de derechos humanos en el diseño de los mismos.

Durante ese mismo periodo, el gobierno nacional adscribió a una política activa a favor de la reivindicación de los derechos humanos, tanto en cuanto a crímenes pasados de lesa humanidad, como a la incorporación del lenguaje de derechos en las normas y en la retórica del discurso político. No resulta casual entonces que leyes, decretos y resoluciones ministeriales encuentren en su contenido el lenguaje de derechos humanos.

Existía una necesidad latente de reconocer la problemática de los jóvenes que ni estudian ni están empleados en la Argentina. ${ }^{16}$ El grupo que más sufre esta problemática son aquellos jóvenes entre 18 y 24 años que pertenecen al quintil más bajo de ingresos (Marzonetto y Aguirre, 2014). Es en este grupo etario donde es más notable la alta desocupación y la precariedad laboral. Pero los problemas son más profundos aún: existe un desajuste entre las competencias adquiridas en la escuela secundaria y la arquitectura institucional del mercado de trabajo. Es por ello que son más propensos a "encontrar empleo de baja calificación, normalmente asociado a sectores informales, con baja competitividad y pocas expectativas de desarrollo futuro" (Marzonetto y Aguirre 2014:10).

El PROGRESAR nace en este contexto, buscando proveer de cobertura social a una población desprovista de protección luego de cumplidos los 18 años. De esta manera se intenta hacer frente a la problemática de la desocupación y la deserción mediante un estipendio mensual que incentive a los jóvenes a retomar los estudios o terminarlos mediante condicionalidades de cursado, asistencia y chequeos médicos anuales. No queda claro, sin embargo, cómo se lograrán los objetivos de inserción laboral y terminalidad educativa teniendo en cuenta las deficiencias en capacidades estatales ${ }^{17}$ así como así también las problemáticas educativas antes mencionadas.

Problema. Toda política pública nace como respuesta a un problema determinado que pretende resolver. En el caso de los $\mathrm{Ni}-\mathrm{Ni}$, organizaciones no gubernamentales, investigadores y gobiernos identifican a este grupo de personas como aquellos jóvenes entre 18-24 años ${ }^{18}$ que se encuentran fuera del sistema educativo y que no tienen empleo y lo están buscando (activos), o que no tienen empleo ni lo buscan (inactivos). Las razones por las que se da este fenómeno y por el cual algún joven sería considerado parte de la clasificación Ni-Ni son varias, entre las que se pueden incluir: la deserción escolar debido a la baja calidad educativa 
que no asegura empleos de calidad; el ejercicio de tareas de cuidado y domésticas que no son remuneradas ni consideradas trabajo formal; el empleo temprano y precario, pero informal, temporario y muchas veces no remunerado; la estructura general del mercado de trabajo que demanda ciertas calificaciones que son inasibles para muchos jóvenes, en especial aquellos en condición de vulnerabilidad; los problemas endémicos ligados al déficit de infraestructura social que no logran "incluir" debidamente a los destinatarios de la política; los problemas de capacidades estatales, específicamente los que hacen al financiamiento de la política.

Ante la situación descripta, el gobierno nacional propuso dar soluciones mediante un programa de transferencias condicionadas que promueva la continuidad escolar y/o el mejoramiento de las calificaciones de los jóvenes a la hora de ingresar al mercado laboral. Ya se señaló que estos objetivos parecieran difíciles de cumplir si sólo se incentiva la demanda y no la oferta en infraestructura social, o no se soluciona la falta de articulación con otros programas tanto a nivel nacional como provincial. Es decir, cumplir con los objetivos del programa demandaría inversión en otros programas paralelos, como por ejemplo el programa "Creciendo Juntos", abocado al cuidado de menores en Argentina, o el plan FINES, diseñado para lograr la terminalidad educativa, que dejó de existir en $2016^{19}$.

Actores, constelaciones y modos de interacción. Cada actor posee percepciones, preferencias y capacidades. Pero resulta poco probable que un actor o grupo de actores logre decisiones de política pública sólo basados en las percepciones y preferencias y mediante el uso de sus capacidades. Es por ello que es importante tener en cuenta la constelación en la que distintos actores intervienen en las interacciones de política pública. Las constelaciones incluyen a los actores, sus estrategias, los posibles resultados de estas estrategias y las preferencias de los actores ante estos resultados. Los modos de interacción denotan las formas específicas de acción en las que actores actúan en determinadas constelaciones situadas en ciertos marcos institucionales (Scharpf, 1997). ${ }^{20}$

Determinados el contexto de la política, el problema y el marco institucional, podemos avanzar en identificar los elementos que restan. Empero, es necesario precisar que en el caso del PROGRESAR no es posible explicar los resultados de una política mediante la teoría de los juegos á la Scharpf porque no existen realmente puntos de conflicto entre los actores que sean claramente identificables, como el autor indica en el caso de gremios y gobiernos. Ante la ausencia de conflicto identificable, resulta también problemático identificar las estrategias y preferencias de los actores en juego, especialmente cuando nos referimos a los beneficiarios. Al parecer, en este caso se trata más bien de un grupo de actores (el gobierno nacional) que condiciona el comportamiento de destinatarios mediante una política pública determinada. El PROGRESAR proviene de una decisión unilateral del ejecutivo nacional. Si bien había una necesidad latente de incluir a un grupo social previamente excluido, y que por razones de edad ya no gozaba de beneficios, no existieron grupos de presión, ni manifestaciones públicas, ni tampoco un llamado de algún sector 
de la sociedad para implementar una política de esta índole. Más bien, pareciera ser que la decisión política de implementar el programa, cual como ocurrió con crisis anteriores, se produjo como forma de amortizar la devaluación producida meses antes. ${ }^{21}$

Mediante el uso del recurso del Decreto de Necesidad y Urgencia (DNU) el gobierno evitó la intermediación del Congreso, legislando y ejecutando a la vez, y conectando en forma directa al decisor de la política con el destinatario. Es así que a un grupo que previamente no había sido interpelado se le otorga rango de actor social, aunque su atomización y la falta de identidad como actor social le previene la capacidad de hacer valer este nuevo derecho de manera efectiva, ya sea mediante la protesta social o recurriendo a la justicia. El hecho de que la prestación no se haya actualizado en casi dos años da cuenta de esto.A su vez, un marco institucional endeble afecta contra la sostenibilidad del programa, dinamitando la posibilidad de que estos derechos se asimilen con el correr del tiempo.Al depender del Poder Ejecutivo Nacional, se corre el riesgo de que un gobierno con distintos objetivos políticos pueda desfinanciar o fragmentar el programa, como efectivamente ocurrió a partir de 2015 con varios programas sociales de menor envergadura e impacto mediático, incluido el mismo PROGRESAR. Así, el modo unilateral de interacción del gobierno atenta con la supervivencia de la política a largo plazo. El resultado es una política desarticulada, desfinanciada y no sustentable, carente de principios claves para que pueda ser considerada una política social con enfoque de derechos.

\section{Conclusiones}

Se intentó aquí hacer un análisis exploratorio de un programa social bajo un marco de análisis novedoso. El mismo intenta analizar políticas sociales con enfoque de derechos desde el institucionalismo centrado en actores. Si bien no todas las categorías desarrolladas por este último enfoque pudieron ser extrapoladas, el marco general sirvió de referencia para analizar la política, las causas de su desarrollo y sus posibles resultados. Sin duda, este enfoque debería ser mejorado y puesto a prueba con otros programas sociales similares en pos de verificar su poder explicativo.

A su vez, un estudio empírico de corte cualitativo coadyuvará a otorgar de mayor precisión a los argumentos aquí expuestos. Es crucial para el éxito de este enfoque poder entender de manera cabal las orientaciones, preferencias y percepciones de los actores, tanto de los decisores como de los destinatarios del programa. Quizás el valor de este trabajo yazca en abrir la discusión de utilizar enfoques provenientes de otras disciplinas y combinarlos para lograr nuevas formas de analizar políticas, intentando superar las limitaciones y riesgos que esta empresa conlleva. 


\begin{abstract}
${ }^{1}$ Este ensayo se respalda en trabajos anteriores.Véase Olmedo (2015,2016,2017).Varias de las ideas presentadas aquí son producto de intercambios con Consuelo Parmigiani de Barbará y Marcelo Nazareno, a quienes agradezco sus aportes. ${ }^{2}$ El autor plantea que muchos académicos aplaudimos la idea de combinar teorías distintas. Esta combinación de teorías y conceptos generarían nuevas perspectivas y agendas de investigación. Ahora bien, el inconveniente radica en armonizar las diferencias ontológicas, epistemológicas y metodológicas entre distintos enfoques. Su solución es dividir esta posible combinación teórica en tres abordajes: síntesis, que produce una teoría basada en los insights de varias teorías; complementario, que usa varias teorías para producir un rango de explicaciones; y contradictoria, que compara los conceptos y explicaciones de varias teorías para luego elegir una sobre las demás (Cairney, 2013). Nuestra propuesta se basa en la segunda de estas opciones.

${ }^{3}$ Olmedo (2017) explora cómo ciertas políticas sociales similares en fechas de iniciación, objetivos, diseño e implementación, adoptan por un lado el enfoque de derechos, como es el caso del PROGRESAR, y por el otro, un enfoque más bien top-down de la política, como es el caso del Programa Primer Paso Aprendiz en la Provincia de Córdoba. ${ }^{4}$ Distintos autores reconocen numerosas variantes dentro del nuevo institucionalismo. Para citar algunas, Peters (2003) las divide en seis categorías distintas, entre ellas el institucionalismo normativo, el de la elección racional, el histórico, el empírico, el internacional y el social. Otros autores (Hall y Taylor, 1996), diferencian tres tipos de institucionalismo: el histórico, el de la elección racional y el sociológico. Powell y Dimaggio (1999) comparten esta división, pero consideran al sociológico dentro de la variante del institucionalismo histórico. Siguiendo una conceptualización similar, Parmigiani de Barbará (2014) reduce las corrientes institucionales a dos: el de la elección racional y el histórico-sociológico. Una casi idéntica clasificación hace Zurbriggen (2006) cuando diferencia entre el institucionalismo racionalista (que abarca el de la elección racional) y el culturalista (que incluye el histórico y el sociológico centrado en la estructura). Este trabajo adhiere a esta última clasificación. Tanto Peters (2003) como Zurbriggen (2006) colocan al institucionalismo centrado en actores (Scharpf, 1997) dentro del de la elección racional.

${ }^{5}$ Traducción del autor

${ }^{6}$ Peters (2003) distingue tres "manifestaciones" dentro de la corriente de la elección racional: los modelos de instituciones basados en la relación jefe-agente, los que están basados en las reglamentaciones y aquellos basados en la teoría de los juegos. En esta última clasificación se encuentra Scharpf (1997).

${ }^{7}$ Ejemplos de sanciones ante la violación de reglas sociales son la pérdida de la reputación, la desaprobación social, la falta de cooperación y recompensas, e incluso el ostracismo (Scharpf, 1997).

${ }^{8}$ En varios pasajes, Scharpf (1997) propone que los buenos marcos institucionales son conducentes a seguir los preceptos de justicia distributiva. Así, el autor considera que la noción de Pareto superior (en la cual una política es aceptable si la ganancia de algunos agentes no perjudican al resto de la sociedad) ha primado sobre el utilitarismo en el diseño de las políticas públicas.

${ }^{9}$ Esto es lo que denominamos como lógica situacional.

${ }^{10}$ Cabe destacar que existen varios autores (Peters, 1999; Acuña, 2014; Shepsle, 2005; Hall y Taylor, 1996, Immergut, 1998; Scharpf, 1997) que explícita o implícitamente pregonan por una convergencia entre las distintas corrientes institucionalistas en pos de lograr un marco teórico común. Sin embargo, hay ciertos supuestos dentro de cada corriente que resultan irreconciliables; específicamente, el deductivismo de la elección racional versus el inductivismo del institucionalismo histórico.

${ }^{11}$ Los elementos constitutivos de este enfoque son los siguientes:
\end{abstract}

- Actores: poseen capacidades (recursos de acción) y percepciones y preferencias (recursos de orientación). Estos a su vez están influidos y limitados por el contexto institucional.

- Constelaciones de actores: describe a los jugadores, sus estrategias, los resultados asociados a esas estrategias, y las preferencias de los actores ante estos resultados. Cualquier constelación puede ser "jugada" en una variedad de modos de interacción: juegos cooperativos o no cooperativos, juegos jerárquicos o juegos de votación.

- Modos de interacción: son formas de interacción que unen el comportamiento de los actores en determinadas constelaciones con marcos institucionales específicos.

- Marcos institucionales: son las reglas que moldean el comportamiento de los actores (con sus preferencias, percepciones y capacidades), los modos de interacción y los resultados deseables. 
${ }^{12}$ Siguiendo la hipótesis de Avelino y otros (2005), el resurgimiento y afianzamiento de gobiernos democráticos en la mayoría de los países latinoamericanos en las dos últimas décadas del siglo pasado contribuyó a la expansión del gasto social, especialmente aquel que está orientado al incremento de capital humano.

${ }^{13}$ Véase el trabajo de María Ester Rosas (2007) sobre trabajo infantil y transferencias monetarias condicionadas en Argentina.

${ }^{14}$ Cabe destacar que los estipendios no se han actualizado desde marzo de 2015, donde la inflación durante ese periodo supera el $70 \%$. Actualmente los beneficiarios cobran $\$ 900$ por mes, un $20 \%$ del cual se liquida a fin de año luego de que el destinatario demuestre que las condicionalidades de estudio (asistencia o cursado) y salud (chequeo médico anual) han sido cumplimentados.

${ }^{15}$ En julio de 2015, el Congreo Nacional aprueba la Ley 27.160 que determina la movilidad de la AUH, la asignación por embarazo y las asignaciones familiares. En ella, se determina que los beneficios serán ajustados de forma semestral, siguiendo el mismo cálculo con el que se actualizan los haberes jubilatorios. El mismo texto contempla un incremento del 30 por zona desfavorable para los residentes de la región patagónica. En abril de 2016, mediante el decreto 593/2016, se amplía la cobertura de las asignaciones familiares al incorporar a los descendientes de monotrobutistas.

${ }^{16}$ Cabe aclarar que muchos de estos jóvenes sí trabajan, ya sea en el sector informal o en tareas de cuidado. Sin embargo se considera que la gran mayoría sufre de dos problemas que están interrelacionados: la deserción escolar y el desempleo.

${ }^{17}$ Las capacidades estatales pueden definirse como la aptitud que tiene el Estado de gestionar e implementar sus políticas públicas. Podemos dividir las capacidades estatales en burocracia y financiamiento. El PROGRESAR presenta deficiencias en ambos componentes por dos razones. Por un lado, hay una ausencia de coordinación interorganizacional entre los distintos organismos del Estado, que permita la articulación entre distintas áreas para lograr los objetivos propuestos por la política (por ejemplo inserción laboral o terminalidad educativa). Es visible a su vez la ausencia de infraestructura social (psicopedagogos, coordinadores, consejeros, etc) que ayuden al destinatario a maximizar su pertenencia en el programa. Por otro lado, pero íntimamente relacionado con lo anterior, se encuentra el financiamiento. El monto del estipendio del PROGRESAR, si bien depende del Tesoro, no se actualiza automáticamente, quedando a merced del gobierno de turno la actualización del beneficio y la sustentabilidad del programa a futuro. Asimismo, el incentivo hacia la demanda, es decir, en estipendios para los beneficiarios, produce un desbalance si no se compensa la infraestructura material y social, obstaculizando la capacidad real del Estado de cumplimentar los objetivos del programa.

${ }^{18}$ En el Reino Unido, se considera Ni-Ni a aquellos jóvenes entre 16 y 24 años. En Canadá o Australia, se extiende desde los 15 a los 29. En Argentina, se considera a un joven Ni-Ni desde los 18 a los 24 años, aunque ciertas jurisdicciones como Córdoba reduce la edad a los 16 años.

${ }^{19}$ El programa Creciendo Juntos, que implementa el Ministerio de Educación en conjunto con el Ministerio de Desarrollo Social de la Nación, tiene lugar en los Centros de Desarrollo Infantil Comunitarios en todo el país. Estos centros asisten en las tareas de cuidado, preparación para la escuela y fortalecimiento de las familias. El programa Creciendo Juntos realiza cursos de formación y capacitación destinado a educadores, madres, cocineras, etc. con el fin de promover el desarrollo de niños y niñas hasta los cuatro años de edad. El programa de Finalización de Estudios Primarios y Secundarios (FINES) fue concebido por el Ministerio de Educación y el Ministerio de Desarrollo Social en el 2008 con el objetivo de lograr la terminalidad educativa de aquellos estudiantes de nivel primario y secundario que sean mayores de 18 años y hayan permanecido fuera de la escuela por más de un año. El programa cuenta con dos modalidades, Trayecto y Deudores, orientado a quienes deseen completar sus estudios primarios/secundarios o rendir materias adeudadas, respectivamente. Similar a lo que sucedió con el PROGRESAR, con el cambio de gobierno nacional a partir de diciembre de 2015, ambos programas sufrieron recortes en sus presupuestos y subsecuentemente en la cantidad de destinatarios, suspensiones en su aplicación y una eventual transferencia de competencias a las jurisdicciones provinciales.

${ }^{20}$ Scharpf (1997) toma la teoría de los juegos para ilustrar su teoría. Así se puede clasificar un problema específico de política pública en juegos teórico-descriptivos, como el del Dilema del Prisionero o el de la Gallina. Los modos de interacción actuarían como las posibles estrategias de acción de las constelaciones de actores, las cuales están influidos por el marco institucional y el contexto de la política.

${ }^{21}$ En enero 2014 el gobierno nacional devaluó la moneda un $22 \%$. 


\section{Referencias bibliográficas}

Abramovich, Victor. (2006): “Una aproximación al enfoque de derechos en las estrategias y políticas de desarrollo”. Revista de la CEPAL 88: 35-50.

Abramovich,Victor. (2013): "La igualdad en las políticas sociales. Retóricas presentes obligaciones pendientes". Ciencias Sociales (Revista de la Facultad de Ciencias Sociales, UBA 84:64-76.

Abramovich, Victor. y C. Courtis (2002): Los derechos sociales como derechos exigibles. Madrid:Trotta.

Abramovich,Victor. y C. Courtis (2006): El umbral de la ciudadanía. Buenos Aires: Ed. del Puerto.

Abramovich, Victor. y Laura Pautassi (2006): Dilemas actuales en la resolución de la pobreza. El aporte del enfoque de derechos. Ponencia presentada en el Seminario "Los Derechos Humanos y las políticas públicas para enfrentar la pobreza y la desigualdad", Buenos Aires, UNESCO, Secretaría de Derechos Humanos y Universidad Nacional Tres de Febrero.

Abramovich, Victor. y Laura Pautassi (2009): "El enfoque de derechos y la institucionalidad de las políticas sociales”. En La revisión judicial de las políticas sociales. Estudio de casos. Ed. del Puerto, Buenos Aires.

Arcidiácono, Pilar (2011):"Inclusión Social, Enfoque de Derechos y Políticas de Transferencias de Ingresos”. En Argentina: ¿Una Combinación Posible? Revista Electrónica del instituto de investigaciones "Ambrosio L. Gioja” - AñoV, Número Especial. Disponible en http:// www.derecho.uba.ar/revistagioja/articulo_esp.php?ided=6\&idsec=14\&art=115

Avelino, G., Brown, D. S. and Hunter, W. (2005), “The Effects of Capital Mobility, Trade Openness, and Democracy on Social Spending in Latin America, 1980-1999". American Journal of Political Science, 49: 625-641

Barrientos, A. y J. M. Villa (2014): "Economic and political inclusion of human development conditional cash transfer programmes in Latin America?”, Working Paper 200, Manchester: Brooks World Poverty Institute.

Bastagli, F. (2009): "From social safety net to social policy? The role of conditional cash transfers in welfare state development in Latin America", Working Paper 60 International Policy Centre for Inclusive Growth.

Boudon, R. (2003): “Beyond Rational Choice Theory”, Annual Review of Sociology Vol. 29:1-21 
Cacace, A. y P. Albornoz (2012): "Fiscalización de los planes sociales en Argentina" Auditoría General de la Nación. Buenos Aires.

Cairney, Paul (2013): "Standing on the Shoulders of Giants: How Do We Combine the Insights of Multiple Theories in Public Policy Studies?” Policy Studies Journal 41: 1-21.

CELS, Centro de Estudios Legales y Sociales (2007): "Programa Familias por la inclusión social. Entre el discurso de derecho y la práctica asistencial". Cuadernos de Investigación y Análisis N² . Buenos Aires, Centro de Estudios Legales y Sociales.

Decreto 593/2016. Boletín Oficial de la República Argentina. Buenos Aires, 19 de abril de 2016

Decreto 90/2018. Boletín Oficial de la República Argentina.Buenos Aires, 31 de enero de 2018

Etchichury, H. y M. Álvarez (2015): Encuentros y Desencuentros Entre las Políticas públicas y los Derechos Sociales. Córdoba: CIJS y GIIDES.

Fiszbein, Ariel, Norbert Schady et al. (2009): Conditional Cash Transfers Reducing Present and Future Poverty. Washington DC: TheWorld Bank.

GTPSS (2015): "Indicadores de Progreso para la Medición de Derechos Contemplados en el Protocolo de San Salvador", elaborado por Grupo de Trabajo del Protocolo de San Salvador. Washington, DC: OEA.

Hall, P. A. \& R.C.R. Taylor (1996): "Political Science and the Three New Institutionalisms". Political Studies 44: 936-957.

Hornes, M. (2013): "Los programas de transferencias monetarias condicionadas. Una aproximación desde la socio-antropología económica”. Revista Debate Público Reflexión de Trabajo Social, Año 3 (5):103-112.

Immergut, E.M. (1998): "The theoretical core of the new institutionalism". Politics \& Society 26 (1), 5-34.

Jones, Bryan D. (1999): “Bounded Rationality”. Annual Review of Political Science,Vol. 2 (1) 297-321.

Levy, S. \& N. Schady (2013): “Latin America's social policy challenge: education, social insurance, redistribution”. Journal of Economic Perspectives 27 (2):193-218.

Ley 27.160. Movilidad de las Asignaciones Familiares. Boletín Oficial de la República Argentina. Buenos Aires, 15 de julio de 2015 
Ley 27.160. Movilidad de las Asignaciones Familiares. Boletín Oficial de la República Argentina. Buenos Aires, 15 de julio de 2015

López-Calva, Felipe y Nora Lustig, eds. (2010) Declining Inequality in Latin America: A Decade of Progress? Washington, DC:The Bookings Institution.

Marzonetto, G. y J. Aguirre (2014): "Sin estudio y sin trabajo. El Programa de Respaldo a Estudiantes Argentinos: PROG.R.ES.AR”. Buenos Aires: CIEPP, Serie Análisis de Coyuntura.

Olmedo, Gonzalo (2015) “¿Beneficiarios o ciudadanos incluidos? Políticas de Inclusión Educativa a Través de Programas de Transferencias Monetarias Condicionales: el PNBE y el PROGRESAR". Ponencia presentada en elVI Coloquio de IIFAP, Córdoba, Argentina.

Olmedo, Gonzalo (2016): “El PROGRESAR desde el Enfoque de Derechos”. Ponencia presentada en el XIII Congreso Nacional de Derecho Político "La Política y el Derecho en el Bicentenario de la Independencia”, Rosario, Argentina.

Olmedo, Gonzalo (2017): "EL PROG.R.ES.AR. desde el enfoque de Derechos Humanos". Revista Administración Pública y Sociedad. 2:24-37.

Ostrom, Elinor (1990) Governing the Commons: The Evolution of Institutions for Collective Action. New York: Cambridge University Press.

Pautassi, Laura (2009): “Programas de transferencias condicionadas de ingresos ¿Quién pensó en el cuidado? La experiencia en Argentina” en Seminario Regional Las familias latinoamericanas interrogadas. Hacia la articulación del diagnóstico, la legislación y las políticas CEPAL, Santiago de Chile.

Pautassi, Laura (2013): “La igualdad en las políticas sociales. Retóricas presentes, obligaciones pendientes". Ciencias Sociales. Revista de la Facultad de Ciencias Sociales, UBA 84:64-69.

Peters, Guy (2003): El nuevo institucionalismo. Teoría institucional en ciencia política. Barcelona: Gedisa.

Poder Ejecutivo Nacional (2014) Decreto 84/2014. Boletín Oficial de la República Argentina, 27 de enero.

Rawlings, L. y G. Rubio (2005) "Evaluating the Impact of Conditional Cash Transfer Programs”. TheWorld Bank Research Working Papers 3119. Washington DC:The World Bank. 
Repetto, Fabian (2009): “El marco institucional de las políticas sociales: Posibilidades y restricciones para la gestión social”. En Nagdalena Chiara y María Mercedes deVirgilio, comps., Gestión de la política socia: conceptos y herramientas. Buenos Aires: Prometeo, cap. 5.

Rodríguez Enríquez, C. (2008) "Programas de transferencias condicionadas de ingreso e igualdad de género ¿Por dónde anda América Latina?” Asuntos de Género109. Santiago de Chile, CEPAL.

Rosas, M.E. (2007): “Trabajo infantil y Programas de Transferencias en Efectivo Condicionadas en Argentina”. Ministerio de Trabajo y Seguridad Social Argentina.

Roth Deubel, A.N. (2008): "Perspectivas teóricas para el análisis de las políticas públicas: ¿de la razón científica al arte retórico?” Estudios Políticos, 33, Instituto de Estudios Políticos, Universidad de Antioquia, 67-91.

Shepsle, K. (2005): "Rational Choice Institutionalism” en Binder S. et al., eds. Oxford Handbook of Political Institutions. Oxford UK: Oxford University Press, cap. 2.

Scharpf, F.W. (1997): Games Real Actors Play: Actor-centered institutionalism in policy research. Boulder, Colorado: Westview Press.

Simon, Herbert (1957): “A Behavioral Model of Rational Choice”, en Models of Man, Social and Rational: Mathematical Essays on Rational Human Behavior in a Social Setting. New York:Wiley \& Sons.

Sotomayor, A. (2008): "Los métodos cualitativos en la ciencia política contemporánea: avances, agendas y retos”. Política y Gobierno 15:159-179.

Villatoro, P. (2007): “Las transferencias condicionadas en América Latina: luces y sombras". Documento para el Seminario Internacional "Evolución y desafios de los programas de transferencias condicionadas". Santiago de Chile: CEPAL.

Zibecchi, Clara (2008): “Programas de transferencia de ingresos. ¿Más condicionalidades y menos derechos para madres pobres?”. Revista Aportes Andinos 21.

Zurbriggen, C. (2006):“El Institucionalismo Centrado En Los Actores: Una perspectiva analítica en el estudio de las políticas públicas”. Revista de Ciencia Política (Santiago de Chile), 26(1), 67-83.

\section{Cómo citar este artículo}

Olmedo, Gonzalo (2018) “Aunando Enfoques Teóricos: el Enfoque de Derechos y el Institucionalismo Centrado en Actores”. Revista Perspectivas de Políticas Públicas vol. 7 No14:393-414 